\title{
OPTICAL RESONANCE OF A THREE-LEVEL SYSTEM IN SEMICONDUCTOR QUANTUM DOTS
}

\author{
Nguyen Van Hieu* \\ Max-Planck Institute for the Physics of Complex Systems, \\ D-01187 Dresden, Germany \\ Institute of Materials Science, VAST and College of Technology VNUH, \\ Hanoi, Vietnam \\ Nguyen Bich Ha \\ Institute of Materials Science, VAST and College of Technology VNUH, \\ Hanoi, Vietnam
}

Received 12 May 2006

\begin{abstract}
The optical resonance of a three-level system of the strongly correlated electrons in the twolevel semiconductor quantum dot interacting with the linearly polarized monochromatic electromagnetic radiation is studied. With the application of the Green function method the expressions of the state vectors and the energies of the stationary states of the system in the regime of the optical resonance are derived. The Rabi oscillations of the electron populations at different levels as well as the Rabi splitting of the peaks in the photon emission spectra are investigated.
\end{abstract}

PACS numbers: 71.35.-y, 78.55.-m, 78.67.Hc

Keywords: quantum dot, optical resonance, Rabi oscillation, population flopping

\section{INTRODUCTION}

The electronic structure of semiconductor quantum dots (QDs) and the electromagnetic interaction processes of the strongly correlated electron systems in these nanostructures were widely investigated. In many theoretical and experimental works the formation and the radiative recombination of the excitons and the biexcitons as well as the electron-electron interactions, including the exchange interaction, in the direct band gap semiconductor QD were studied. If between two states of the electron system in the QD the radiative transitions are allowed then at the electromagnetic radiation frequency in the range of the resonance with these transitions the optical resonance phenomenon with the Rabi oscillations of the populations of these states occurs, as in the case of the optical resonance of the two-level atomic systems [1]. The Rabi oscillations in the semiconductor QDs were studied in many experimental and theoretical works [2 - 18]. The Rabi oscillations in the four-level double structures were recently investigated [19,

${ }^{*}$ Corresponding author e-mail: nvhieu@iop.vast.ac.vn 
20]. The vacuum Rabi splitting of the exciton in the semiconductor QD interacting with the quantized electromagnetic field in a microcavity was also widely studied [21]. The photon absorption induced electron transport through semiconductor QDs is the basics of the physics of QD photodetectors [17, 18, 22]. A photodetector is most sensible when the frequency of the radiation is in the resonance with the dipole transition between the ground state of the QD and the exciton state. In this frequency range the Rabi oscillations play a major role in the generation of the photocurrent.

The simplest model of the semiconductor QD's for the study of the optical resonance as well as the photon absorption induced electron transport would be the disk-shaped direct band gap semiconductor QD with two discrete energy levels, the upper level being that of an electron from the conduction band in the confining potential field of the fabricated QD, while the lower one being that of an electron from the heavy-hole valence band $\left(J=\frac{3}{2}\right.$ and $\left.J_{z}= \pm \frac{3}{2}\right)$. We call it shortly the two-level semiconductor QD. Even in this simplest semiconductor QD the electron system cannot be always considered as the analogy of a two- level atomic system containing only one electron, because the QD may contain not only one electron, but also two or three electrons. For example, the linearly polarized monochromatic electromagnetic radiation with the frequency in the range of the optical resonance generates the Rabi oscillations between the ground state of the QD (that without any electron and any hole) and some exciton state as well as between this exciton state and the biexciton one. These three states form a three-level system and there must be some influence of the biexciton on the Rabi oscillations between the ground state and the exciton state, as this was discussed by many authors [2, 7, 8, 12, 18]. The twophoton Rabi oscillations of the biexciton were observed in a recent experimental work [14]. The theory of the Rabi oscillations of the three-level system "ground state-exciton-biexciton" in the two-level disk-shaped semiconductor QD interacting with the linearly polarized monochromatic electromagnetic radiation will be presented in this work.

In the theory of the optical resonance in a two-level atomic system the optical Bloch equation was established and analytically solved [1]. Similarly, for the study on the Rabi oscillations in semiconductors the semiconductor Bloch equations [23 - 31] together with their approximate and numerical solutions were used. In the present work instead of using the semiconductor Bloch equations for the QD we shall apply the Green function technique.

In Sec. 2 the eigenstates and the corresponding eigenvalues of the Hamiltonian of the Hubbard type of the electron system in a two-level semiconductor QD with the strong Coulomb interaction between the electrons as well as with their anisotropic exchange interaction will be given. It will be shown that beside of several pairs of eigenstates which can be considered as the analogies of a two-level atomic system there exist also a triplet of three eigenstates which must be considered as a three-level system. The Green function technique for the study of the Rabi oscillations in this three-level system will be presented in Sec. 3. The analytical expressions of the Green functions are derived exactly, all Rabi oscillations are found and the algebraic equations determining the Rabi frequencies are established. In particular, the influence of the biexciton state on the optical resonance between the ground state of the QD and the exciton state as well as the two-photon Rabi oscillations of the biexciton will be investigated in details. The Rabi flopping of the populations between different levels of the electron system and the structure of the photon emission spectrum as well as the polarization properties of the emitted photons are studied in Sec. 4 and Sec. 5. We use the unit system with $\hbar=c=1$.

\section{STATE VECTORS AND ENERGY SPECTRUM OF THE ELECTRON SYSTEM}

We consider the simplest model of the semiconductor QD with two discrete energy levels $E_{1}^{0}$ (in the conduction band) and $E_{2}^{0}$ (in the heavy-hole valence band). Denote $c_{i \sigma}$ and 162 
$c_{i \sigma}^{+}, i=1,2$, the annihilation and creation operators of the electrons with the spin projection $\sigma=\uparrow, \downarrow$ at these levels and assume the Hubbard form expression of the Hamiltonian of the electron system in the QD:

$$
\begin{array}{r}
H_{d o t}=\sum_{i=1,2}\left[E_{i}^{0} c_{i}^{+} c_{i}+U_{i} n_{i \uparrow} n_{i \downarrow}\right]+U_{12} n_{1} n_{2}+U_{e x} s_{1 z} s_{2 z}+V_{e x}\left[s_{1 x} s_{2 x}+s_{1 y} s_{2 y}\right], \\
c_{i}=\left(\begin{array}{c}
c_{i \uparrow} \\
c_{i \downarrow}
\end{array}\right), E_{1}^{0}>E_{2}^{0}, n_{i \sigma}=c_{i \sigma}^{+} c_{i \sigma}, n_{i}=n_{i \uparrow}+n_{i \downarrow}, s_{i \alpha}=\frac{1}{2} c_{i}^{+} \sigma_{\alpha} c_{i},
\end{array}
$$

$\alpha=x, y, z, \sigma_{x}, \sigma_{y}, \sigma_{z}$ being the Pauli matrices. In the formula (1) $U_{1}, U_{2}$ and $U_{12}$ are the potential energies of the Coulomb interaction between two electrons at the same energy level $E_{1}$, $E_{2}$ or at two different ones, $U_{e x}$ and $V_{e x}$ are two constants of the exchange interaction between two electrons at different levels. We assume the approximate cylindrical symmetry with the symmetry axis $\mathrm{O} z$ so that there are two exchange interaction constants $U_{e x}$ and $V_{e x}$. For the definiteness we chose the periodic Bloch factors in the wave functions of the electrons in the heavy-hole band to be $\left|\frac{X+i Y}{\sqrt{2}} \uparrow\right\rangle$ and $\left|\frac{X-i Y}{\sqrt{2}} \downarrow\right\rangle$.

In the QD there exist 16 different states of the electron system:

- One state without any electron at both levels - the vacuum state $|0\rangle$ with the vanishing energy. In the electron-hole formalism it is the state of two holes.

- Four one-electron states $\Phi_{i}^{\sigma}=c_{i \sigma}^{+}|0\rangle, \sigma=\uparrow, \downarrow, i=1,2$. In the electron-hole formalism $\Phi_{1}^{\sigma}$ are two states of the positive trion - the exciton-hole complex, $\Phi_{2}^{\sigma}$ are two states of the hole.

- Six two-electron states $\Phi_{11}=c_{1 \uparrow}^{+} c_{1 \downarrow}^{+}|0\rangle, \quad \Phi_{22}=c_{2 \uparrow}^{+} c_{2 \downarrow}^{+}|0\rangle, \quad \Phi_{12}^{\sigma}=c_{1 \sigma}^{+} c_{2 \sigma}^{+}|0\rangle, \quad \sigma=\uparrow, \downarrow$, $\Phi_{12}^{t}=\frac{1}{\sqrt{2}}\left(c_{1 \uparrow}^{+} c_{2 \downarrow}^{+}+c_{1 \downarrow}^{+} c_{2 \uparrow}^{+}\right)|0\rangle, \quad \Phi_{12}^{s}=\frac{1}{\sqrt{2}}\left(c_{1 \uparrow}^{+} c_{2 \downarrow}^{+}-c_{1 \downarrow}^{+} c_{2 \uparrow}^{+}\right)|0\rangle$.

In the electron-hole formalism $\Phi_{22}$ is the state without any electron and any hole - the ground state of the QD, $\Phi_{11}$ is that of the biexciton, $\Phi_{12}^{\sigma}, \sigma=\uparrow, \downarrow$ and $\Phi_{12}^{t}$ are three states of the triplet exciton, $\Phi_{12}^{s}$ is that of the singlet exciton.

- Four three-electron states $\Phi_{112}^{\sigma}=c_{1 \uparrow}^{+} c_{1 \downarrow}^{+} c_{2 \sigma}^{+}|0\rangle, \Phi_{122}^{\sigma}=c_{1 \sigma}^{+} c_{2 \uparrow}^{+} c_{2 \downarrow}^{+}|0\rangle, \sigma=\uparrow, \downarrow$. In the electron-hole formalism $\Phi_{112}^{\sigma}$ are two states of the negative trion - the exciton-electron complex, $\Phi_{122}^{\sigma}$ are two states of one electron at the energy level in the conduction band and without any hole.

- One four-electron state $\Phi_{1122}=c_{1 \uparrow}^{+} c_{1 \downarrow}^{+} c_{2 \uparrow}^{+} c_{2 \downarrow}^{+}|0\rangle$. In the electron-hole formalism it is the state without any hole and with two electrons.

In the presence of the interaction between the electrons in the QD and the monochromatic electromagnetic wave having the frequency $\omega_{0}$ in the resonance with the radiative transitions between two discrete energy levels the stationary states of the electron system in the QD must be changed due to the appearance of the Rabi oscillations. Since we are interested only in the 
resonant radiative transitions we shall neglect the terms in the interaction Hamiltonian which give no contribution to these resonant transitions. This is the rotating wave approximation (RWA).

The concrete expression of the interaction Hamiltonian depends on the polarization properties of the electromagnetic radiation. We shall study the two-level QD interacting with the electromagnetic radiation linearly polarized in the direction of the axis $\mathrm{O} x$. In this case the matrix elements of the transitions

$$
c_{1 \sigma} \leftrightarrow c_{2 \sigma}, \sigma=\uparrow, \downarrow,
$$

do not depend on the spin projections of the electrons. In the RWA the interaction Hamiltonian has the form

$$
H_{e m}=\lambda\left(e^{i \omega_{0} t} c_{2}^{+} c_{1}+e^{-i \omega_{0} t} c_{1}^{+} c_{2}\right) .
$$

The total Hamiltonian of the QD interacting with the electromagnetic radiation equals

$$
H=H_{d o t}+H_{e m} .
$$

Following the earlier work [22] we eliminate the explicitly time-dependent factors $e^{ \pm i \omega_{0} t}$ in the interaction Hamiltonian by means of the unitary transformation

$$
\begin{gathered}
U=e^{i \frac{\omega_{0} t}{2}\left(c_{2}^{+} c_{2}-c_{1}^{+} c_{1}\right)}, \\
\tilde{H}_{d o t}=U^{+} H_{d o t} U+i \frac{d U^{+}}{d t} U, \\
\tilde{H}=U^{+} H U+i \frac{d U^{+}}{d t} U,
\end{gathered}
$$

and use a new representation in which the total Hamiltonian is time-independent

$$
\begin{aligned}
\tilde{H}=\sum_{i=1,2}\left[E_{i} c_{i}^{+} c_{i}\right. & \left.+U_{i} n_{i \uparrow} n_{i \downarrow}\right]+U_{12} n_{1} n_{2}+U_{e x} s_{1 z} s_{2 z}+ \\
& +V_{e x}\left[s_{1 x} s_{2 x}+s_{1 y} s_{2 y}\right]+\lambda\left(c_{2}^{+} c_{1}+c_{1}^{+} c_{2}\right),
\end{aligned}
$$

where

$$
E_{1}=E_{1}^{0}-\frac{\omega_{0}}{2}, \quad E_{2}=E_{2}^{0}+\frac{\omega_{0}}{2} .
$$

For the definiteness we chose $\omega_{0} \leq E_{1}^{0}-E_{2}^{0}$ so that $E_{1} \geq E_{2}$. The Hamiltonian (7) generates the mixing between following eigenstates of the Hamiltonian (5):

$$
\Phi_{1}^{\sigma} \leftrightarrow \Phi_{2}^{\sigma}, \Phi_{11} \leftrightarrow \Phi_{12}^{s} \leftrightarrow \Phi_{22}, \Phi_{112}^{\sigma} \leftrightarrow \Phi_{122}^{\sigma}, \sigma=\uparrow, \downarrow .
$$

Therefore two pairs of one-electron states and two pairs of three-electron states are four twolevel systems, while the set of three two-electron states $\Phi_{11}, \Phi_{12}^{s}$ and $\Phi_{22}$ is a three-level system. The separate two-electron state $\Phi_{12}^{t}$ does not participate in the Rabi oscillations.

Note that because of the exclusion Pauli principle in the transitions between the three-electron states $\Phi_{112}^{\sigma} \leftrightarrow \Phi_{122}^{\sigma}$ there are the transitions of only the electron with the spin projection $-\sigma$ 
between two discrete energy levels $c_{1-\sigma}^{+} \leftrightarrow c_{2-\sigma}^{+}$. Therefore the Rabi frequency of the optical resonance in the two-level system of three-electron states is the same as that of the one-electron states.

\section{GREEN FUNCTIONS AND RABI OSCILATIONS}

For the study of the optical resonance of the systems of two-electron states in the QD we introduce the two-particle Green functions

$$
G^{i j k l}\left(t-t^{\prime}\right)=-i \theta\left(t-t^{\prime}\right)\left\langle 0\left|c_{i \sigma}(t) c_{j-\sigma}(t) c_{k-\sigma}^{+}\left(t^{\prime}\right) c_{l \sigma}^{+}\left(t^{\prime}\right)\right| 0\right\rangle,
$$

$i, j, k, l=1$, 2, with some fixed $\sigma=\uparrow$ or $\downarrow$, and denote $F^{i j k l}(\omega)$ their Fourier transforms:

$$
G^{i j k l}\left(t-t^{\prime}\right)=\frac{1}{2 \pi} \int e^{-i \omega\left(t-t^{\prime}\right)} F^{i j k l}(\omega) d \omega .
$$

The system of differential equations for the Green functions (9) was derived with the use of the total Hamiltonian (7) and the corresponding system of algebraic equations for the Fourier transforms $F^{i j k l}(\omega)$ was solved. These functions $F^{i j k l}(\omega)$ contain three roots $\widetilde{E}_{11}, \widetilde{E}_{12}$ and $\widetilde{E}_{22}$ of the algebraic equation

$$
\left(\omega-E_{11}\right)\left(\omega-E_{12}^{s}\right)\left(\omega-E_{22}\right)-2 \lambda^{2}\left(2 \omega-E_{11}-E_{22}\right)=0 .
$$

where

$$
E_{11}=E_{11}^{0}-\omega_{0}, \quad E_{22}=E_{22}^{0}+\omega_{0}
$$

$E_{11}^{0}, E_{12}^{s}$ and $E_{22}^{0}$ being the eigenvalues of the Hamiltonian (1) corresponding to its eigenstates $\Phi_{11}, \Phi_{12}^{s}$ and $\Phi_{22}$ :

$$
E_{11}^{0}=2 E_{1}^{0}+U_{1}, \quad E_{22}^{0}=2 E_{2}^{0}+U_{2}, E_{12}^{s}=E_{1}^{0}+E_{2}^{0}+U_{12}-\frac{1}{4} U_{e x}-\frac{1}{2} V_{e x} .
$$

We chose the notations such that

$$
\tilde{E}_{11} \rightarrow E_{11}, \quad \tilde{E}_{12} \rightarrow E_{12}^{s}, \quad \tilde{E}_{22} \rightarrow E_{22} \text { at } \lambda \rightarrow 0
$$

and have

$$
\begin{aligned}
F^{1111}(\omega)= & \frac{\left(\widetilde{E}_{11}-E_{12}^{s}\right)\left(\widetilde{E}_{11}-E_{22}\right)-2 \lambda^{2}}{\left(\widetilde{E}_{11}-\widetilde{E}_{12}\right)\left(\widetilde{E}_{11}-\widetilde{E}_{22}\right)} \frac{1}{\omega-\widetilde{E}_{11}} \\
& +\frac{\left(\widetilde{E}_{12}-E_{12}^{s}\right)\left(\widetilde{E}_{12}-E_{22}\right)-2 \lambda^{2}}{\left(\widetilde{E}_{12}-\widetilde{E}_{11}\right)\left(\widetilde{E}_{12}-\widetilde{E}_{22}\right)} \frac{1}{\omega-\widetilde{E}_{12}} \\
& +\frac{\left(\widetilde{E}_{22}-E_{12}^{s}\right)\left(\widetilde{E}_{22}-E_{22}\right)-2 \lambda^{2}}{\left(\widetilde{E}_{22}-\widetilde{E}_{11}\right)\left(\widetilde{E}_{22}-\widetilde{E}_{12}\right)} \frac{1}{\omega-\widetilde{E}_{22}},
\end{aligned}
$$




$$
\begin{aligned}
& F^{2222}(\omega)=\frac{\left(\widetilde{E}_{11}-E_{11}\right)\left(\widetilde{E}_{11}-E_{12}^{s}\right)-2 \lambda^{2}}{\left(\widetilde{E}_{11}-\widetilde{E}_{12}\right)\left(\widetilde{E}_{11}-\widetilde{E}_{22}\right)} \cdot \frac{1}{\omega-\widetilde{E}_{11}} \\
& +\frac{\left(\widetilde{E}_{12}-E_{11}\right)\left(\widetilde{E}_{12}-E_{12}^{s}\right)-2 \lambda^{2}}{\left(\widetilde{E}_{12}-\widetilde{E}_{11}\right)\left(\widetilde{E}_{12}-\widetilde{E}_{22}\right)} \cdot \frac{1}{\omega-\widetilde{E}_{12}} \\
& +\frac{\left(\widetilde{E}_{22}-E_{11}\right)\left(\widetilde{E}_{22}-E_{12}^{s}\right)-2 \lambda^{2}}{\left(\widetilde{E}_{22}-\widetilde{E}_{11}\right)\left(\widetilde{E}_{22}-\widetilde{E}_{12}\right)} \cdot \frac{1}{\omega-\widetilde{E}_{22}}, \\
& F^{1122}(\omega)=F^{2211}(\omega)=\frac{2 \lambda^{2}}{\left(\widetilde{E}_{11}-\widetilde{E}_{12}\right)\left(\widetilde{E}_{11}-\widetilde{E}_{22}\right)} \frac{1}{\omega-\widetilde{E}_{11}} \\
& +\frac{2 \lambda^{2}}{\left(\widetilde{E}_{12}-\widetilde{E}_{11}\right)\left(\widetilde{E}_{12}-\widetilde{E}_{22}\right)} \frac{1}{\omega-\widetilde{E}_{12}} \\
& +\frac{2 \lambda^{2}}{\left(\widetilde{E}_{22}-\widetilde{E}_{11}\right)\left(\widetilde{E}_{22}-\widetilde{E}_{12}\right)} \frac{1}{\omega-\widetilde{E}_{22}}, \\
& F^{1212}(\omega)+F^{1221}(\omega)=F^{2112}(\omega)+F^{2121}(\omega)= \\
& =F^{1212}(\omega)+F^{2112}(\omega)=F^{1221}(\omega)+F^{2121}(\omega)= \\
& =\frac{\left(\widetilde{E}_{11}-E_{11}\right)\left(\widetilde{E}_{11}-E_{22}\right)}{\left(\widetilde{E}_{11}-\widetilde{E}_{12}\right)\left(\widetilde{E}_{11}-\widetilde{E}_{22}\right)} \frac{1}{\omega-\widetilde{E}_{11}}+\frac{\left(\widetilde{E}_{12}-E_{11}\right)\left(\widetilde{E}_{12}-E_{22}\right)}{\left(\widetilde{E}_{12}-\widetilde{E}_{11}\right)\left(\widetilde{E}_{12}-\widetilde{E}_{22}\right)} \frac{1}{\omega-\widetilde{E}_{12}} \\
& +\frac{\left(\widetilde{E}_{22}-E_{11}\right)\left(\widetilde{E}_{22}-E_{22}\right)}{\left(\widetilde{E}_{22}-\widetilde{E}_{11}\right)\left(\widetilde{E}_{22}-\widetilde{E}_{12}\right)} \frac{1}{\omega-\widetilde{E}_{22}}, \\
& F^{1211}(\omega)=F^{2111}(\omega)=F^{1112}(\omega)=F^{1121}(\omega)= \\
& =\lambda \frac{\widetilde{E}_{11}-E_{22}}{\left(\widetilde{E}_{11}-\widetilde{E}_{12}\right)\left(\widetilde{E}_{11}-\widetilde{E}_{22}\right)} \frac{1}{\omega-\widetilde{E}_{11}}+\lambda \frac{\widetilde{E}_{12}-E_{22}}{\left(\widetilde{E}_{12}-\widetilde{E}_{11}\right)\left(\widetilde{E}_{12}-\widetilde{E}_{22}\right)} \frac{1}{\omega-\widetilde{E}_{12}} \\
& +\lambda \frac{\widetilde{E}_{22}-E_{22}}{\left(\widetilde{E}_{22}-\widetilde{E}_{11}\right)\left(\widetilde{E}_{22}-\widetilde{E}_{12}\right)} \frac{1}{\omega-\widetilde{E}_{22}}, \\
& F^{1222}(\omega)=F^{2122}(\omega)=F^{2212}(\omega)=F^{2221}(\omega)= \\
& =\lambda \frac{\widetilde{E}_{11}-E_{11}}{\left(\widetilde{E}_{11}-\widetilde{E}_{12}\right)\left(\widetilde{E}_{11}-\widetilde{E}_{22}\right)} \frac{1}{\omega-\widetilde{E}_{11}}+\lambda \frac{\widetilde{E}_{12}-E_{11}}{\left(\widetilde{E}_{12}-\widetilde{E}_{11}\right)\left(\widetilde{E}_{12}-\widetilde{E}_{22}\right)} \frac{1}{\omega-\widetilde{E}_{12}} \\
& +\lambda \frac{\widetilde{E}_{22}-E_{11}}{\left(\widetilde{E}_{22}-\widetilde{E}_{11}\right)\left(\widetilde{E}_{22}-\widetilde{E}_{12}\right)} \frac{1}{\omega-\widetilde{E}_{22}} \cdot
\end{aligned}
$$


The poles $\widetilde{E}_{11}, \widetilde{E}_{12}$ and $\widetilde{E}_{22}$ in the expressions (12) - (17) of the Fourier transforms of the twoparticle Green functions are the eigenvalues of the total Hamiltonian (7). Denote $\widetilde{\Phi}_{11}, \widetilde{\Phi}_{12}$ and $\widetilde{\Phi}_{22}$ the corresponding eigenstates of this Hamiltonian,

$$
\tilde{\Phi}_{11} \rightarrow \Phi_{11}, \tilde{\Phi}_{12} \rightarrow \Phi_{12}^{s}, \tilde{\Phi}_{22} \rightarrow \Phi_{22} \text { at } \lambda \rightarrow 0 .
$$

The eigenstates $\Phi_{11}, \Phi_{12}^{s}$ and $\Phi_{22}$ of the Hamiltonian (5) of the electron system in the QD in the absence of the interaction with the electromagnetic radiation are three linear combinations of them

$$
\begin{aligned}
& \Phi_{11}=a_{11} \widetilde{\Phi}_{11}+a_{12} \widetilde{\Phi}_{12}+a_{13} \widetilde{\Phi}_{22}, \\
& \Phi_{12}^{s}=a_{21} \tilde{\Phi}_{11}+a_{22} \widetilde{\Phi}_{12}+a_{23} \widetilde{\Phi}_{22}, \\
& \Phi_{22}=a_{31} \widetilde{\Phi}_{11}+a_{32} \widetilde{\Phi}_{12}+a_{33} \widetilde{\Phi}_{22} .
\end{aligned}
$$

Calculating the Fourier transforms of the two-particle Green functions (9) with the use of the relations (18) and comparing the results with the expressions (12)-(17) we obtain

$$
\begin{aligned}
& a_{11}^{2}=\frac{\left(\widetilde{E}_{11}-E_{12}^{s}\right)\left(\widetilde{E}_{11}-E_{22}\right)-2 \lambda^{2}}{\left(\widetilde{E}_{11}-\widetilde{E}_{12}\right)\left(\widetilde{E}_{11}-\widetilde{E}_{22}\right)} \quad, \quad a_{12}^{2}=\frac{\left(\widetilde{E}_{12}-E_{12}^{s}\right)\left(\widetilde{E}_{12}-E_{22}\right)-2 \lambda^{2}}{\left(\widetilde{E}_{12}-\widetilde{E}_{11}\right)\left(\widetilde{E}_{12}-\widetilde{E}_{22}\right)} \text {, } \\
& a_{13}^{2}=\frac{\left(\widetilde{E}_{22}-E_{12}^{s}\right)\left(\widetilde{E}_{22}-E_{22}\right)-2 \lambda^{2}}{\left(\widetilde{E}_{22}-\widetilde{E}_{11}\right)\left(\widetilde{E}_{22}-\widetilde{E}_{12}\right)} ; \\
& a_{21}^{2}=\frac{\left(\widetilde{E}_{11}-E_{11}\right)\left(\widetilde{E}_{11}-E_{22}\right)}{\left(\widetilde{E}_{11}-\widetilde{E}_{12}\right)\left(\widetilde{E}_{11}-\widetilde{E}_{22}\right)} \quad, \quad a_{22}^{2}=\frac{\left(\widetilde{E}_{12}-E_{11}\right)\left(\widetilde{E}_{12}-E_{22}\right)}{\left(\widetilde{E}_{12}-\widetilde{E}_{11}\right)\left(\widetilde{E}_{12}-\widetilde{E}_{22}\right)}, \\
& a_{23}^{2}=\frac{\left(\widetilde{E}_{22}-E_{11}\right)\left(\widetilde{E}_{22}-E_{22}\right)}{\left(\widetilde{E}_{22}-\widetilde{E}_{11}\right)\left(\widetilde{E}_{22}-\widetilde{E}_{12}\right)} \\
& a_{31}^{2}=\frac{\left(\tilde{E}_{11}-E_{11}\right)\left(\tilde{E}_{11}-E_{12}^{s}\right)-2 \lambda^{2}}{\left(\widetilde{E}_{11}-\widetilde{E}_{12}\right)\left(\widetilde{E}_{11}-\widetilde{E}_{22}\right)} \quad, \quad a_{32}^{2}=\frac{\left(\widetilde{E}_{12}-E_{11}\right)\left(\tilde{E}_{12}-E_{12}^{s}\right)-2 \lambda^{2}}{\left(\widetilde{E}_{12}-\widetilde{E}_{11}\right)\left(\widetilde{E}_{12}-\widetilde{E}_{22}\right)}, \\
& a_{33}^{2}=\frac{\left(\widetilde{E}_{22}-E_{11}\right)\left(\widetilde{E}_{22}-E_{12}^{s}\right)-2 \lambda^{2}}{\left(\widetilde{E}_{22}-\widetilde{E}_{11}\right)\left(\widetilde{E}_{22}-\widetilde{E}_{12}\right)} ; \\
& a_{11} a_{21}=\sqrt{2} \lambda \frac{\widetilde{E}_{11}-E_{22}}{\left(\widetilde{E}_{11}-\widetilde{E}_{12}\right)\left(\widetilde{E}_{11}-\widetilde{E}_{22}\right)}, a_{12} a_{22}=\sqrt{2} \lambda \frac{\widetilde{E}_{12}-E_{22}}{\left(\widetilde{E}_{12}-\widetilde{E}_{11}\right)\left(\widetilde{E}_{12}-\widetilde{E}_{22}\right)}, \\
& a_{13} a_{23}=\sqrt{2} \lambda \frac{\widetilde{E}_{22}-E_{22}}{\left(\widetilde{E}_{22}-\widetilde{E}_{11}\right)\left(\widetilde{E}_{22}-\widetilde{E}_{12}\right)} ;
\end{aligned}
$$




$$
\begin{gathered}
a_{11} a_{31}=\frac{2 \lambda^{2}}{\left(\widetilde{E}_{11}-\widetilde{E}_{12}\right)\left(\widetilde{E}_{11}-\widetilde{E}_{22}\right)} \quad, \quad a_{12} a_{32}=\frac{2 \lambda^{2}}{\left(\widetilde{E}_{12}-\widetilde{E}_{11}\right)\left(\widetilde{E}_{12}-\widetilde{E}_{22}\right)}, \\
a_{13} a_{33}=\frac{2 \lambda^{2}}{\left(\widetilde{E}_{22}-\widetilde{E}_{11}\right)\left(\widetilde{E}_{22}-\widetilde{E}_{12}\right)} ; \\
a_{21} a_{31}=\sqrt{2} \lambda \frac{\widetilde{E}_{11}-E_{11}}{\left(\widetilde{E}_{11}-\widetilde{E}_{12}\right)\left(\widetilde{E}_{11}-\widetilde{E}_{22}\right)}, a_{22} a_{32}=\sqrt{2} \lambda \frac{\widetilde{E}_{12}-E_{11}}{\left(\widetilde{E}_{12}-\widetilde{E}_{11}\right)\left(\widetilde{E}_{12}-\widetilde{E}_{22}\right)}, \\
a_{23} a_{33}=\sqrt{2} \lambda \frac{\widetilde{E}_{22}-E_{11}}{\left(\widetilde{E}_{22}-\widetilde{E}_{11}\right)\left(\widetilde{E}_{22}-\widetilde{E}_{12}\right)} \cdot
\end{gathered}
$$

The coefficients $a_{\alpha \beta}$ satisfy following relations

$$
\begin{gathered}
a_{11}^{2}+a_{12}^{2}+a_{13}^{2}=a_{21}^{2}+a_{22}^{2}+a_{23}^{2}=a_{31}^{2}+a_{32}^{2}+a_{33}^{2}=1, \\
a_{11} a_{21}+a_{12} a_{22}+a_{13} a_{23}=a_{11} a_{31}+a_{12} a_{32}+a_{13} a_{33}=a_{21} a_{31}+a_{22} a_{32}+a_{23} a_{33}=0 .
\end{gathered}
$$

This means that the linear transformation (18) is unitary and has following inverse transformation

$$
\begin{aligned}
& \widetilde{\Phi}_{11}=a_{11} \Phi_{11}+a_{21} \Phi_{12}^{s}+a_{31} \Phi_{22}, \\
& \widetilde{\Phi}_{12}=a_{12} \Phi_{11}+a_{22} \Phi_{12}^{s}+a_{32} \Phi_{22}, \\
& \widetilde{\Phi}_{22}=a_{13} \Phi_{11}+a_{23} \Phi_{12}^{s}+a_{33} \Phi_{22} .
\end{aligned}
$$

The formulae (18) - (24) and (26) would be needed for the study of the resonant photon emission from the QD in the optical resonant regime as well as the photon absorption induced electron transport through the QD.

Usually the biexciton has a non-vanishing binding energy. Therefore, three types of radiative transitions $\Phi_{22} \leftrightarrow \Phi_{12}^{s}, \Phi_{12}^{s} \leftrightarrow \Phi_{11}$ and $\Phi_{22} \leftrightarrow \Phi_{11}$ cannot be simultaneously at the resonance: if one type of transition is at the resonance, then the others are only near the resonance. For the study of the Rabi oscillations between the ground state of the QD and the singlet exciton state we consider the special case when the radiation frequency $\omega_{0}$ is tuned to the value at the resonance with the transitions between them:

$$
\omega_{0}=E_{12}^{s}-E_{22}^{0} \text {. }
$$

In this case

$$
E_{12}^{s}=E_{22}
$$

and the equation (11) becomes

$$
\left(\omega-E_{22}\right)^{2}\left(\omega-E_{11}\right)-2 \lambda^{2}\left(2 \omega-E_{11}-E_{22}\right)=0 .
$$


Denote $2 \delta$ the binding energy of the biexciton,

$$
\delta=E_{12}^{s}-\frac{E_{11}+E_{22}}{2},
$$

and suppose that

$$
\left|\frac{\lambda}{\delta}\right|<<1 .
$$

If the terms of the order $\lambda / \delta$ and higher are neglected then equation (27) has following three approximate roots

$$
\widetilde{E}_{11} \approx E_{11}, \widetilde{E}_{12} \approx E_{22}+\sqrt{2}|\lambda|, \tilde{E}_{22} \approx E_{22}-\sqrt{2}|\lambda|,
$$

and from the expressions (19)-(24) we obtain following approximate values of the coefficients in the transformation (18):

$$
a_{11} \approx 1, a_{12} \approx a_{21} \approx a_{13} \approx a_{31} \approx 0, a_{22} \approx a_{33} \approx \frac{1}{\sqrt{2}},-a_{23} \approx a_{32} \approx \frac{1}{\sqrt{2}} \frac{\lambda}{|\lambda|} .
$$

The formulae (28) and (29) show that if the small terms of the order $\lambda / \delta$ and higher are neglected then the pair of the ground state of the QD and the singlet exciton state behaves at the resonance like a two-level atomic system but with the Rabi frequency

$$
\Delta \approx 2 \sqrt{2}|\lambda|,
$$

which is $\sqrt{2}$ times larger than the value of the Rabi frequency of the two-level system of oneelectron state $\Phi_{1}^{\sigma}$ and $\Phi_{2}^{\sigma}$ (with a given spin projection $\sigma$ ). That is because the linearly polarized electromagnetic radiation induces the resonant transitions of both electrons in the twoelectron states and therefore the intensity of the transitions between the two-electron states $\Phi_{22} \leftrightarrow \Phi_{12}^{s}$ is twice of that of the transitions between the one-electron states $\Phi_{1}^{\sigma} \leftrightarrow \Phi_{2}^{\sigma}$.

However, in the first order with respect to the ratios $\lambda / \delta$ instead of the formulae (28) we have

$$
\widetilde{E}_{11} \approx E_{11}-\frac{\lambda^{2}}{\delta}, \quad \widetilde{E}_{12} \approx E_{22}+\sqrt{2}|\lambda|+\frac{\lambda^{2}}{2 \delta}, \quad \widetilde{E}_{22} \approx E_{22}-\sqrt{2}|\lambda|+\frac{\lambda^{2}}{2 \delta},
$$

and instead of the formulae (29) we have

$$
\begin{aligned}
& a_{11} \approx 1, \quad a_{12} \approx \frac{\lambda}{2 \delta}, \quad a_{13} \approx-\frac{|\lambda|}{2 \delta}, \quad a_{21} \approx-\frac{\lambda}{\sqrt{2} \delta}, \quad a_{31} \approx 0, \\
& a_{22} \approx a_{33} \approx \frac{1}{\sqrt{2}}\left(1+\frac{|\lambda|}{4 \sqrt{2} \delta}\right), \quad-a_{23} \approx a_{32} \approx \frac{1}{\sqrt{2}} \frac{\lambda}{|\lambda|}\left(1-\frac{|\lambda|}{4 \sqrt{2} \delta}\right) .
\end{aligned}
$$

The formulae (31) show that in this order there are two different Rabi frequencies $2 \sqrt{2}|\lambda| \pm \lambda^{2} / \delta$. In comparison with the formulae (28) and (29) the new terms of the order $\lambda / \delta$ in the formulae (31) and (32) determine the influence of the biexciton on the resonant Rabi 
oscillations between the singlet exciton and the ground state of the QD. This influence is significant if $\lambda$ and $\delta$ have the magnitudes of the same order.

Now we consider another special case: the resonant two-photon transitions $\Phi_{22} \leftrightarrow \Phi_{11}$ between the ground state of the QD and that of the biexciton [14], when the radiation frequency $\omega_{0}$ is tuned to the value

$$
\omega_{0}=\frac{E_{11}^{0}-E_{22}^{0}}{2} \quad, \quad E_{11}=E_{22}=E_{0}
$$

Equation (11) becomes

$$
\left(\omega-E_{0}\right)\left[\left(\omega-E_{0}\right)\left(\omega-E_{12}^{s}\right)-4 \lambda^{2}\right]=0
$$

and is exactly solvable. The assignments of its roots with the eigenvalue $\widetilde{E}_{11}, \widetilde{E}_{12}$ and $\widetilde{E}_{22}$ corresponding to the eigenstates $\widetilde{\Phi}_{11}, \widetilde{\Phi}_{12}$ and $\widetilde{\Phi}_{22}$ of the total Hamiltonian (7) depend on the sign of $\delta$. We have

$$
\begin{aligned}
& \widetilde{E}_{11}=E_{0}, \widetilde{E}_{12}=\frac{E_{0}+E_{12}^{s}}{2}+\frac{1}{2} \Delta, \widetilde{E}_{22}=\frac{E_{0}+E_{12}^{s}}{2}-\frac{1}{2} \Delta \text { if } \delta>0, \\
& \widetilde{E}_{11}=\frac{E_{0}+E_{12}^{s}}{2}+\frac{1}{2} \Delta, \widetilde{E}_{12}=\frac{E_{0}+E_{12}^{s}}{2}-\frac{1}{2} \Delta, \widetilde{E}_{22}=E_{0} \text { if } \delta<0,
\end{aligned}
$$

where

$$
\Delta=\left[\left(E_{0}-E_{12}^{s}\right)^{2}+16 \lambda^{2}\right]^{1 / 2}
$$

is the Rabi frequency at the resonance. From the formulae (34) or (35) for the eigenvalues $\widetilde{E}_{11}, \widetilde{E}_{12}$ and $\widetilde{E}_{22}$ of the total Hamiltonian (7) and the relations (19)-(24) we obtain following expressions of the coefficients $a_{\alpha \beta}, \alpha, \beta=1,2,3$, of the unitary transformation (18) in the case $\delta>0$ :

$$
\begin{array}{lll}
a_{11}=\frac{1}{\sqrt{2}} \quad, \quad a_{12}=\frac{2 \lambda}{\sqrt{\Delta(\Delta+\delta)}} \quad, \quad a_{13}=\frac{2 \lambda}{\sqrt{\Delta(\Delta-\delta)}}, \\
a_{21}=0 \quad, \quad a_{22}=\frac{1}{\sqrt{2}} \sqrt{\frac{\Delta+\delta}{\Delta}} \quad, \quad a_{23}=-\frac{1}{\sqrt{2} \sqrt{\frac{\Delta-\delta}{\Delta}}}, \\
a_{31}=-\frac{1}{\sqrt{2}} \quad, \quad a_{32}=\frac{2 \lambda}{\sqrt{\Delta(\Delta+\delta)}}, \quad a_{33}=\frac{2 \lambda}{\sqrt{\Delta(\Delta-\delta)}} .
\end{array}
$$

The formulae in the case $\delta<0$ can be derived in a similar manner.

It is interesting to consider the QD with the vanishing biexciton binding energy:

$$
E_{12}^{s}=\frac{E_{11}+E_{22}}{2}=E_{0} \text {. }
$$

In this case the equation (11) becomes 170 


$$
\left(\omega-E_{0}\right)\left[\left(\omega-E_{11}\right)\left(\omega-E_{22}\right)-\lambda^{2}\right]=0
$$

with the roots

$$
\widetilde{E}_{11}=E_{0}, \widetilde{E}_{12}=E_{0}+\frac{1}{2} \Omega, \widetilde{E}_{22}=E_{0}-\frac{1}{2} \Omega,
$$

where $\Omega$ is the Rabi frequency

$$
\Omega=\left[\left(E_{11}-E_{22}\right)^{2}+16 \lambda^{2}\right]^{1 / 2} .
$$

For the definiteness we suppose that $E_{11} \geq E_{22}$. Then

$$
\tilde{E}_{11} \rightarrow E_{11}, \tilde{E}_{22} \rightarrow E_{22} \text { at } \lambda \rightarrow 0 .
$$

At the resonance $E_{11}=E_{22}$ the Rabi frequency $\Omega$ becomes

$$
\Delta=4|\lambda|
$$

and the matrix elements $a_{\alpha \beta}$ of the unitary transformation (18) equal

$$
a_{11}=a_{22}=-a_{23}=-a_{31}=\frac{1}{\sqrt{2}}, a_{21}=0, a_{12}=a_{13}=a_{32}=a_{33}=\frac{1}{2} \text {. }
$$

This means that in the case of the QD with the vanishing biexciton binding energy the radiation frequency $\omega_{0}$ can be tuned to the resonance with both types of radiative transitions $\Phi_{22} \leftrightarrow \Phi_{12}^{s}$ , $\Phi_{12}^{s} \leftrightarrow \Phi_{11}$ as well as to the resonance with the two-photon transitions $\Phi_{22} \leftrightarrow \Phi_{11}$ and therefore there exist all three types of level mixings: between the QD ground state and the singlet exciton, between the singlet exciton and the biexciton, and also between the QD ground state and the biexciton. Note that the resonance Rabi frequency (41) of the three-level system of two-electron states $\Phi_{11}, \Phi_{12}^{s}$ and $\Phi_{22}$ is twice of that of the two-level system of one-electron states.

It can be shown that if the biexciton binding energy is very small and the radiation frequency $\omega_{0}$ is very near to the resonances with both types of radiative transitions $\Phi_{22} \leftrightarrow \Phi_{12}^{s}$ and $\Phi_{12}^{s} \leftrightarrow \Phi_{11}$, then the simultaneous mixing between all three states also takes place - the role of the biexciton on the optical resonance of the QD is significant.

\section{RABI FLOPPING OF POPULATIONS}

Due to the optical resonance in the QD there appears the Rabi flopping between its different energy levels. Suppose that at the initial time moment $t=0$ both states with two spin projections $\sigma=\uparrow, \downarrow$ at the lower energy level $E_{2}^{0}$ are occupied by the electrons, while the upper energy level $E_{1}^{0}$ is empty. Then at a later time moment $t>0$ the mean value of the number of the electrons at the upper level equals

$$
\bar{n}_{1}(t)=\left\langle\Phi_{22}\left|c_{1}^{+}(t) c_{1}(t)\right| \Phi_{22}\right\rangle=\left\langle\Phi_{22}\left|e^{i \tilde{H} t} c_{1}^{+} c_{1} e^{-i \tilde{H} t}\right| \Phi_{22}\right\rangle .
$$


Performing the unitary transformation (18) of $\Phi_{22}$ into a linear combination of $\widetilde{\Phi}_{11}, \widetilde{\Phi}_{12}$, $\widetilde{\Phi}_{22}$, applying the Schrödinger equation

$$
\tilde{H} \tilde{\Phi}_{11}=\widetilde{E}_{11} \widetilde{\Phi}_{11}, \tilde{H} \tilde{\Phi}_{12}=\widetilde{E}_{12} \tilde{\Phi}_{12}, \tilde{H} \tilde{\Phi}_{22}=\widetilde{E}_{22} \tilde{\Phi}_{22}
$$

and then performing again the inverse transformation (26) of $\widetilde{\Phi}_{11}, \widetilde{\Phi}_{12}$ and $\widetilde{\Phi}_{22}$ into the linear combinations of $\Phi_{11}, \Phi_{12}^{s}$ and $\Phi_{22}$, we obtain

$$
\begin{aligned}
\bar{n}_{1}(t)= & a_{31}^{2}\left(a_{21}^{2}+2 a_{11}^{2}\right)+a_{32}^{2}\left(a_{22}^{2}+2 a_{12}^{2}\right)+a_{33}^{2}\left(a_{23}^{2}+2 a_{13}^{2}\right) \\
& +2\left(a_{31} a_{21} a_{32} a_{22}+2 a_{32} a_{12} a_{31} a_{11}\right) \cos \left(\widetilde{E}_{11}-\widetilde{E}_{12}\right) t \\
& +2\left(a_{33} a_{23} a_{31} a_{21}+2 a_{33} a_{13} a_{31} a_{11}\right) \cos \left(\widetilde{E}_{11}-\widetilde{E}_{22}\right) t \\
& +2\left(a_{33} a_{23} a_{32} a_{22}+2 a_{33} a_{13} a_{32} a_{12}\right) \cos \left(\widetilde{E}_{12}-\widetilde{E}_{22}\right) t
\end{aligned}
$$

The expressions of the products of the coefficient $a_{\alpha \beta}$ in the formula (44) were given in Sec.3.

Together with the mean value of the number of the electrons at the upper level $\bar{n}_{1}(t)$ there are also other similar physical quantities characterizing the Rabi population flopping, for example the mean value of the number of the pairs each of which consists of two electrons with both spin projection $\sigma=\uparrow, \downarrow$ at the same upper energy level

$$
n_{11}(t)=\left\langle\Phi_{22}\left|c_{1 \uparrow}^{+}(t) c_{1 \uparrow}(t) c_{1 \downarrow}^{+}(t) c_{1 \downarrow}(t)\right| \Phi_{22}\right\rangle,
$$

and that of the pairs each of which consists of one electron at the upper level and another electron at the lower one

$$
n_{12}(t)=\left\langle\Phi_{22}\left|c_{1}^{+}(t) c_{1}(t) c_{2}^{+}(t) c_{2}(t)\right| \Phi_{22}\right\rangle .
$$

$n_{11}(t)$ is the population of the biexciton state while $n_{12}(t)$ is that of the exciton state. We have

$$
\begin{aligned}
\bar{n}_{11}(t) & =a_{31}^{2} a_{11}^{2}+a_{32}^{2} a_{12}^{2}+a_{33}^{2} a_{13}^{2}+2 a_{32} a_{12} a_{31} a_{11} \cos \left(\widetilde{E}_{11}-\widetilde{E}_{12}\right) t \\
& +2 a_{33} a_{13} a_{31} a_{11} \cos \left(\tilde{E}_{11}-\widetilde{E}_{22}\right) t+2 a_{33} a_{13} a_{32} a_{12} \cos \left(\widetilde{E}_{12}-\widetilde{E}_{22}\right) t .
\end{aligned}
$$

and

$$
\begin{aligned}
\bar{n}_{12}(t) & =a_{31}^{2} a_{21}^{2}+a_{32}^{2} a_{22}^{2}+a_{33}^{2} a_{23}^{2}+2 a_{31} a_{21} a_{32} a_{22} \cos \left(\widetilde{E}_{11}-\widetilde{E}_{12}\right) t \\
& +2 a_{33} a_{23} a_{31} a_{21} \cos \left(\widetilde{E}_{11}-\widetilde{E}_{22}\right) t+2 a_{33} a_{23} a_{32} a_{22} \cos \left(\widetilde{E}_{12}-\widetilde{E}_{22}\right) t .
\end{aligned}
$$

It is easy to see that

$$
\bar{n}_{1}(t)=2 \bar{n}_{11}(t)+\bar{n}_{12}(t) .
$$

The Rabi oscillations of the populations (44), (47) and (48) depend on three frequencies

$$
\Omega^{(1)}=\tilde{E}_{11}-\tilde{E}_{12}, \Omega^{(2)}=\tilde{E}_{12}-\widetilde{E}_{22}, \Omega^{(3)}=\Omega^{(1)}+\Omega^{(2)} .
$$

At the resonance of the two-photon transitions between the ground state of the QD and that of the biexciton [14], $E_{11}=E_{22}$, they are expressed in terms of the Rabi frequency (36) and the constant $\delta$ : 
$\Omega^{(1)}=-\frac{1}{2}(\delta+\Delta), \Omega^{(2)}=\Delta$ if $\delta>0 ; \Omega^{(1)}=\Delta, \Omega^{(2)}=\frac{1}{2}(\delta-\Delta)$ if $\delta<0$.

In the case $\delta>0$ the formulae (44), (47) and (48) become

$$
\begin{gathered}
\bar{n}_{1}(t)=1-\frac{8 \lambda^{2}}{\Delta(\Delta-\delta)} \cos \frac{\Delta-\delta}{2} t-\frac{8 \lambda^{2}}{\Delta(\Delta+\delta)} \cos \frac{\Delta+\delta}{2} t, \\
\bar{n}_{11}(t)=\frac{4 \lambda^{2}}{\Delta(\Delta-\delta)}\left(1-\cos \frac{\Delta-\delta}{2} t\right)+\frac{4 \lambda^{2}}{\Delta(\Delta+\delta)}\left(1-\cos \frac{\Delta+\delta}{2} t\right) \\
-\frac{2 \lambda^{2}}{\Delta^{2}}(1-\cos \Delta t), \\
\bar{n}_{12}(t)=\frac{4 \lambda^{2}}{\Delta^{2}}(1-\cos \Delta t) .
\end{gathered}
$$

In the experiments usually the dependence of the population on the pulse area was measured. From the formulae (45) - (48), (50) - (53) it follows the similar oscillations of this physical observable quantity.

In the special case of the vanishing biexciton binding energy ( $\delta=0$ ) and at the resonance of both types of radiative transitions: between the ground state of the QD and the exciton as well as between the exciton and the biexciton,

$$
E_{12}^{s}=E_{11}=E_{22},
$$

the formulae (51), (52) and (53) have the simple forms

$$
\begin{gathered}
\bar{n}_{1}(t)=1-\cos \frac{1}{2} \Delta t, \\
\bar{n}_{11}(t)=\frac{3}{8}-\frac{1}{2} \cos \frac{1}{2} \Delta t+\frac{1}{8} \cos \Delta t, \\
\bar{n}_{12}(t)=\frac{1}{4}(1-\cos \Delta t),
\end{gathered}
$$

where $\Delta$ is determined by the formula (41).

\section{RABI SPLITTING IN PHOTON EMISSION SPECTRA}

For the study of the spontaneous emission of the photon with a given energy $\omega$ from the QD in the regime of the optical resonance we introduce the effective Hamiltonian of the interaction between electrons and photons. Denote $\gamma_{x}\left(\gamma_{y}\right)$ and $\gamma_{x}^{+}\left(\gamma_{y}^{+}\right)$the annihilation and creation operators for the photon with the energy $\omega$ and the linear polarization along the axis $\mathrm{O} x(\mathrm{O} y)$. With the above- mentioned convention on the electron wave function at the heavy-hole band we have following effective photon-electron interaction Hamiltonian 


$$
H_{\mathrm{int}}=\frac{1}{\sqrt{2}} g\left[\left(\gamma_{x}^{+}-i \gamma_{y}^{+}\right) c_{2 \uparrow}^{+} c_{1 \uparrow}+\left(\gamma_{x}^{+}+i \gamma_{y}^{+}\right) c_{2 \downarrow}^{+} c_{1 \downarrow}\right] e^{i \omega t}+H . c .,
$$

where the effective coupling constant $g$ is proportional to the matrix element of the dipole transition of electrons between two discrete energy levels. The terms not contributing to the photon emission assisting the transition of the electron from the upper level to the lower one as well as to the inverse process are not included in the expression (57).

Between three two-electron states $\widetilde{\Phi}_{11}, \widetilde{\Phi}_{12}$ and $\widetilde{\Phi}_{22}$ there exist 6 photon emission assisted transitions. It can be shown that the photons emitted from all these transitions are linearly polarized along the axis $\mathrm{O} x$. The rates of these transitions and the energies of the emitted photons are

$$
\begin{aligned}
& \left|\left\langle\gamma_{x}, \tilde{\Phi}_{12}\left|H_{\text {int }}\right| \tilde{\Phi}_{11}\right\rangle\right|^{2}=|g|^{2}\left(a_{32} a_{21}+a_{22} a_{11}\right)^{2}, \quad \omega=\omega_{0}+\tilde{E}_{11}-\widetilde{E}_{12}, \\
& \left|\left\langle\gamma_{x}, \tilde{\Phi}_{11}\left|H_{\mathrm{int}}\right| \tilde{\Phi}_{12}\right\rangle\right|^{2}=|g|^{2}\left(a_{31} a_{22}+a_{21} a_{12}\right)^{2}, \quad \omega=\omega_{0}+\tilde{E}_{12}-\widetilde{E}_{11} \text {, } \\
& \left|\left\langle\gamma_{x}, \tilde{\Phi}_{22}\left|H_{\text {int }}\right| \tilde{\Phi}_{12}\right\rangle\right|^{2}=|g|^{2}\left(a_{33} a_{22}+a_{23} a_{12}\right)^{2}, \quad \omega=\omega_{0}+\tilde{E}_{12}-\widetilde{E}_{22} \text {, } \\
& \left|\left\langle\gamma_{x}, \tilde{\Phi}_{12}\left|H_{\mathrm{int}}\right| \tilde{\Phi}_{22}\right\rangle\right|^{2}=|g|^{2}\left(a_{32} a_{23}+a_{22} a_{13}\right)^{2}, \quad \omega=\omega_{0}+\tilde{E}_{22}-\tilde{E}_{12}, \\
& \left|\left\langle\gamma_{x}, \tilde{\Phi}_{22}\left|H_{\mathrm{int}}\right| \tilde{\Phi}_{11}\right\rangle\right|^{2}=|g|^{2}\left(a_{33} a_{21}+a_{23} a_{11}\right)^{2}, \omega=\omega_{0}+\tilde{E}_{11}-\tilde{E}_{22}, \\
& \left|\left\langle\gamma_{x}, \tilde{\Phi}_{11}\left|H_{\mathrm{int}}\right| \tilde{\Phi}_{22}\right\rangle\right|^{2}=|g|^{2}\left(a_{31} a_{23}+a_{21} a_{13}\right)^{2}, \quad \omega=\omega_{0}+\tilde{E}_{22}-\tilde{E}_{11} .
\end{aligned}
$$

Together with six photon emission processes considered above there exist also three photon emission assisted transitions from the states $\widetilde{\Phi}_{11}, \widetilde{\Phi}_{12}$ and $\widetilde{\Phi}_{22}$ to the state $\Phi_{12}^{t}$. It can be shown that the emitted photons are linearly polarized along the axis $\mathrm{O} y$. Their rates and the corresponding photon energies are

$$
\begin{aligned}
& \left|\left\langle\gamma_{y}, \Phi_{12}^{t}\left|H_{\mathrm{int}}\right| \tilde{\Phi}_{11}\right\rangle\right|^{2}=|g|^{2} a_{11}^{2}, \omega=\omega_{0}+\widetilde{E}_{11}-E_{12}^{t}, \\
& \left|\left\langle\gamma_{y}, \Phi_{12}^{t}\left|H_{\mathrm{int}}\right| \tilde{\Phi}_{12}\right\rangle\right|^{2}=|g|^{2} a_{12}^{2}, \omega=\omega_{0}+\widetilde{E}_{12}-E_{12}^{t}, \\
& \left|\left\langle\gamma_{y}, \Phi_{12}^{t}\left|H_{\mathrm{int}}\right| \tilde{\Phi}_{22}\right\rangle\right|^{2}=|g|^{2} a_{13}^{2}, \omega=\omega_{0}+\widetilde{E}_{22}-E_{12}^{t} .
\end{aligned}
$$

The intensities of the spectral lines are proportional to the products of the transition rates and the populations of the corresponding initial states $\left|\tilde{\Phi}_{11}\right\rangle,\left|\tilde{\Phi}_{12}\right\rangle$ and $\left|\tilde{\Phi}_{22}\right\rangle$. Because before the pumping by the resonant electromagnetic radiation the two-electron system exists in the ground state $\left|\Phi_{22}\right\rangle$,

$$
\Phi_{22}=a_{31} \tilde{\Phi}_{11}+a_{32} \tilde{\Phi}_{12}+a_{33} \tilde{\Phi}_{22}
$$


the populations of the initial states $\widetilde{\Phi}_{11}, \widetilde{\Phi}_{12}$ and $\widetilde{\Phi}_{22}$ are $a_{31}^{2}, a_{32}^{2}$ and $a_{33}^{2}$, resp.

Consider now two special cases when the radiation frequency $\omega_{0}$ is tuned to the corresponding resonance values. For the definiteness suppose that the biexciton binding energy is positive, $\delta>0$. In the special case of the resonance between the biexciton and the ground state of the QD from the formulae (34) and (37) we obtain following expressions of the (relative) intensities of nine peaks and the energies of the emitted photons:

$$
\begin{aligned}
& I\left(\tilde{\Phi}_{11} \rightarrow \tilde{\Phi}_{12}\right)=C \frac{\Delta+\delta}{8 \Delta} \quad, \omega=\omega_{0}-\frac{1}{2}(\Delta+\delta), \\
& I\left(\tilde{\Phi}_{12} \rightarrow \tilde{\Phi}_{11}\right)=C \frac{\lambda^{2}}{\Delta^{2}} \quad, \omega=\omega_{0}+\frac{1}{2}(\Delta+\delta), \\
& I\left(\tilde{\Phi}_{12} \rightarrow \tilde{\Phi}_{22}\right)=C \frac{2 \lambda^{2} \delta^{2}}{\Delta^{3}(\Delta+\delta)}, \omega=\omega_{0}+\Delta, \\
& I\left(\tilde{\Phi}_{22} \rightarrow \tilde{\Phi}_{12}\right)=C \frac{2 \lambda^{2} \delta^{2}}{\Delta^{3}(\Delta-\delta)}, \omega=\omega_{0}-\Delta, \\
& I\left(\tilde{\Phi}_{11} \rightarrow \tilde{\Phi}_{22}\right)=C \frac{\Delta-\delta}{8 \Delta}, \omega=\omega_{0}+\frac{1}{2}(\Delta-\delta), \\
& I\left(\tilde{\Phi}_{22} \rightarrow \tilde{\Phi}_{11}\right)=C \frac{\lambda^{2}}{\Delta^{2}}, \omega=\omega_{0}-\frac{1}{2}(\Delta-\delta), \\
& I\left(\tilde{\Phi}_{11} \rightarrow \Phi_{e x}^{t}\right)=C \frac{1}{4}, \omega=\omega_{0}-\delta+E_{e x}^{s}-E_{e x}^{t}, \\
& I\left(\tilde{\Phi}_{12} \rightarrow \Phi_{e x}^{t}\right)=C \frac{16 \lambda^{4}}{\Delta^{2}(\Delta+\delta)^{2}}, \omega=\omega_{0}+\frac{1}{2}(\Delta-\delta)+E_{e x}^{s}-E_{e x}^{t}, \\
& I\left(\tilde{\Phi}_{22} \rightarrow \Phi_{e x}^{t}\right)=C \frac{16 \lambda^{4}}{\Delta^{2}(\Delta-\delta)^{2}}, \omega=\omega_{0}-\frac{1}{2}(\Delta+\delta)+E_{e x}^{s}-E_{e x}^{t}
\end{aligned}
$$

with some constants $C$, the resonance Rabi frequency $\Delta$ being determined by the formula (36). If the constants $\lambda$ and $\delta$ have the magnitudes of the same order, then the rates of all nine abovementioned transitions are comparable.

However, if the radiation frequency $\omega_{0}$ is tuned to the value corresponding to the resonance between the singlet exciton and the ground state of the QD,

$$
E_{12}^{s}=E_{22},
$$

then there appears another spontaneous photon emission spectrum. Suppose that $|\lambda / \delta|<<1$. From the formula (29) it follows that in this special case the transition rates (59), (62), (63), (65) and (66) as well as the population of the initial state $\widetilde{\Phi}_{11}$ of the transitions in the formulae (58) 
and (64) vanish. Therefore in the photon emission spectrum there are only two peaks. Their (relative) intensities and energies of the emitted photons are

$$
\begin{gathered}
I\left(\tilde{\Phi}_{12} \rightarrow \tilde{\Phi}_{22}\right)=C \frac{1}{8} \quad, \omega=\omega_{0}+2 \sqrt{2}|\lambda|, \\
I\left(\tilde{\Phi}_{22} \rightarrow \tilde{\Phi}_{12}\right)=C \frac{1}{8} \quad, \omega=\omega_{0}-2 \sqrt{2}|\lambda| .
\end{gathered}
$$

The damping of the Rabi oscillations due to the electron-phonon interaction was studied in several works $[6,9,11,13,16,18]$. However, the electron-phonon interaction in the QD is not only the origin of the damping of the Rabi oscillations. It might cause also the change of the photon emission spectrum: some spontaneous photon emission processes are suppressed by the fast exciton relaxation and there appear some new ones, because beside of the states $\widetilde{\Phi}_{11}, \widetilde{\Phi}_{12}$ and $\widetilde{\Phi}_{22}$ of the three-level system there exists also the separate triplet exciton state $\Phi_{12}^{t}$ with the energy

$$
E_{12}^{t}=E_{1}^{0}+E_{2}^{0}+U_{12}-\frac{1}{4} U_{e x}+\frac{1}{2} V_{e x} .
$$

Suppose that there is some strong non-radiative phonon assisted relaxation mechanism $\Phi_{12}^{s} \stackrel{\text { phonon }}{\longrightarrow} \Phi_{12}^{t}$. If $\widetilde{E}_{22}<E_{12}^{t}<\widetilde{E}_{12}$, for example, then the radiative transitions $\tilde{\Phi}_{12} \rightarrow \widetilde{\Phi}_{11}, \quad \widetilde{\Phi}_{12} \rightarrow \widetilde{\Phi}_{22}$ and $\tilde{\Phi}_{12} \rightarrow \Phi_{12}^{t}$ with the rates (59), (60) and (65) are suppressed by the phonon-emitting non-radiative fast relaxation $\widetilde{\Phi}_{12} \stackrel{\text { phonon }}{\longrightarrow} \Phi_{12}^{t}$. Similarly, in the case $E_{12}^{t}<\widetilde{E}_{22}\left(<\widetilde{E}_{12}\right)$ the radiative transitions $\widetilde{\Phi}_{12} \rightarrow \widetilde{\Phi}_{11}, \widetilde{\Phi}_{12} \rightarrow \widetilde{\Phi}_{22}, \widetilde{\Phi}_{22} \rightarrow \widetilde{\Phi}_{11}, \widetilde{\Phi}_{22} \rightarrow \widetilde{\Phi}_{12}$, and $\widetilde{\Phi}_{12} \rightarrow \Phi_{12}^{t}, \widetilde{\Phi}_{22} \rightarrow \Phi_{12}^{t}$ with the rates (59), (60), (61), (63) and (65), (66) are suppressed (at the resonance the rate of the non-radiative relaxation $\tilde{\Phi}_{11} \stackrel{\text { phonon }}{\longrightarrow} \Phi_{12}^{t}$ vanishes, because $a_{21}=0$ ). There appear, however, three new photon emission processes $\Phi_{12}^{t} \rightarrow \widetilde{\Phi}_{11}, \Phi_{12}^{t} \rightarrow \widetilde{\Phi}_{12}, \Phi_{12}^{t} \rightarrow \widetilde{\Phi}_{22}$. The emitted photons are linearly polarized along the axis $\mathrm{O} y$. The rates of these transitions and the energies of the emitted photons are

$$
\begin{aligned}
& \left|\left\langle\gamma_{y}, \tilde{\Phi}_{11}\left|H_{\mathrm{int}}\right| \Phi_{12}^{t}\right\rangle\right|^{2}=|g|^{2} a_{31}^{2}, \quad \omega=\omega_{0}+E_{12}^{t}-\widetilde{E}_{11}, \\
& \left|\left\langle\gamma_{y}, \tilde{\Phi}_{12}\left|H_{\mathrm{int}}\right| \Phi_{12}^{t}\right\rangle\right|^{2}=|g|^{2} a_{32}^{2}, \quad \omega=\omega_{0}+E_{12}^{t}-\widetilde{E}_{12}, \\
& \left|\left\langle\gamma_{y}, \tilde{\Phi}_{22}\left|H_{\mathrm{int}}\right| \Phi_{12}^{t}\right\rangle\right|^{2}=|g|^{2} a_{33}^{2}, \quad \omega=\omega_{0}+E_{12}^{t}-\widetilde{E}_{22} .
\end{aligned}
$$

Because the separate state $\Phi_{12}^{t}$ is created by the non-radiative fast relaxation from the state $\widetilde{\Phi}_{12}$ with the population $a_{32}^{2}$ in the case $\widetilde{E}_{22}<E_{12}^{t}<\widetilde{E}_{12}$ and from two states $\widetilde{\Phi}_{12}$ and $\widetilde{\Phi}_{22}$ with the populations $a_{32}^{2}$ and $a_{33}^{2}$ in the case $E_{12}^{t}<\widetilde{E}_{22}\left(<\widetilde{E}_{12}\right)$, the population of the separate state $\Phi_{12}^{t}$ equals $a_{32}^{2}$ in the first case and $a_{32}^{2}+a_{33}^{2}$ in the second case.

If the radiation frequency $\omega_{0}$ is tuned to the value corresponding to the resonance between the biexciton and the ground state of the QD then three new peaks have following (relative) intensities and emitted photon energies: 


$$
\begin{aligned}
& I\left(\Phi_{12}^{t} \rightarrow \tilde{\Phi}_{11}\right)=C^{\prime} \frac{1}{2}, \omega=\omega_{0}+\delta+E_{12}^{t}-E_{12}^{s}, \\
& I\left(\Phi_{12}^{t} \rightarrow \widetilde{\Phi}_{12}\right)=C^{\prime} \frac{4 \lambda^{2}}{\Delta(\Delta+\delta)}, \omega=\omega_{0}-\frac{1}{2}(\Delta-\delta)+E_{12}^{t}-E_{12}^{s}, \\
& I\left(\Phi_{12}^{t} \rightarrow \widetilde{\Phi}_{22}\right)=C^{\prime} \frac{4 \lambda^{2}}{\Delta(\Delta-\delta)}, \omega=\omega_{0}+\frac{1}{2}(\Delta+\delta)+E_{12}^{t}-E_{12}^{s},
\end{aligned}
$$

where

$$
C^{\prime}=\frac{4 \lambda^{2}}{\Delta(\Delta+\delta)} C \text { if } \tilde{E}_{22}<E_{12}^{t}<\widetilde{E}_{12}
$$

and

$$
C^{\prime}=\frac{1}{2} C \text { if } E_{12}^{t}<\tilde{E}_{22}\left(<\tilde{E}_{12}\right) .
$$

However, at the resonance between the singlet exciton and the ground state of the QD in the case $|\lambda / \delta|<<1$ there are only two peaks with following (relative) intensities and emitted photon energies

$$
\begin{aligned}
& I\left(\Phi_{12}^{t} \rightarrow \widetilde{\Phi}_{12}\right)=C^{\prime} \frac{1}{2}, \omega=\omega_{0}+\delta+\sqrt{2}|\lambda|+E_{12}^{t}-E_{12}^{s}, \\
& I\left(\Phi_{12}^{t} \rightarrow \widetilde{\Phi}_{22}\right)=C^{\prime} \frac{1}{2}, \omega=\omega_{0}+\delta-\sqrt{2}|\lambda|+E_{12}^{t}-E_{12}^{s},
\end{aligned}
$$

where

$$
C^{\prime}=\frac{1}{2} C \text { if } \tilde{E}_{22}<E_{12}^{t}<\tilde{E}_{12}
$$

and

$$
C^{\prime}=C \text { if } E_{12}^{t}<\widetilde{E}_{22}\left(<\tilde{E}_{12}\right)
$$

The rate of the transition $\Phi_{12}^{t} \rightarrow \widetilde{\Phi}_{11}$ is very small because $a_{31} \approx 0$.

Note that if $E_{12}^{t}>\widetilde{E}_{12}$, then the above-mentioned suppression of the radiative transitions does not take place.

\section{CONCLUSION AND DISCUSSIONS}

The theory of the Rabi oscillations in the strongly correlated electron system of the two-level semiconductor QD based on the Green function method was presented. The three-level system of the two-electron states in the QD interacting with the linearly polarized electromagnetic radiation was investigated in details. The Rabi flopping of the populations of different states and the Rabi splitting in the photon emission spectra were considered. The structures of the emission spectra depend on the relative magnitudes of the biexciton binding energy $2 \delta$ and the effective 
coupling constant $\lambda$ as well as on the magnitude and the sign of the fine-structure energy splitting constant $E_{12}^{t}-E_{12}^{s}$ of the excitons due to the asymmetry of the QD.

In many experimental works [7, 14, 32 - 34] the QDs with the biexciton binding energies in the range from $2,5 \mathrm{meV}$ to $3,5 \mathrm{meV}$ were investigated. With the intensities of the pumping electromagnetic radiation used for the study of the Rabi oscillations in QDs the coupling constant $\lambda$ has the magnitudes in the range $50-100 \mu \mathrm{eV}$ [3]. Therefore usually we have $|\lambda / \delta|<<1$. On the other hand the magnitudes of the fine-structure energy splitting constants $E_{12}^{t}-E_{12}^{s}$ of the excitons in the QDs investigated in different experiments [2, 8, 33, 34 - 37] have different values in a wide range from $6 \mu \mathrm{eV}$ to $150 \mu \mathrm{eV}$. Note that if we change the linear polarization of the pumping electromagnetic radiation from the direction along the axis $\mathrm{O} x$ to that along the axis $\mathrm{O} y$ then the roles of two exciton states $\Phi_{12}^{s}$ and $\Phi_{12}^{t}$ with the energies $E_{12}^{s}$ and $E_{12}^{t}$ will be interchanged.

In each experiment with the given values of the parameters $E_{11}, E_{12}^{s}, E_{22}, E_{12}^{t}$ and $\lambda$ of the QD and the pumping electromagnetic radiation the positions and the (relative) intensities of the peaks in the spontaneous photon emission spectrum can be determined by the formulae presented in the preceding Section. As the first example consider the two-photon Rabi oscillations of the biexciton in the case $E_{12}^{t}>\widetilde{E}_{12}$. If the biexciton binding energy $2 \delta$ is large, $|\lambda / \delta|<<1$, then in the emission spectrum there are 4 peaks at the energies

$$
\omega_{0}-\Delta, \omega_{0}-\frac{1}{2}(\Delta+\delta), \omega_{0}-\delta+E_{12}^{s}-E_{12}^{t}, \omega_{0}-\frac{1}{2}(\Delta+\delta)+E_{12}^{s}-E_{12}^{t}
$$

with nearly the same intensity $\frac{1}{4} C$, other peaks being very weak. However, if the biexciton has the vanishing binding energy, $\delta=0$, then in the emission spectrum there are five observable peaks: two peaks at the photon energies $\omega_{0} \pm \frac{1}{2} \Delta$ with the same intensity $\frac{3}{16} C$ and the linear polarization along the axis $\mathrm{O} x$, one peak at the photon energy $\omega_{0}+E_{12}^{s}-E_{12}^{t}$ with the intensity $\frac{1}{4} C$ and two peaks at the photon energies $\omega_{0} \pm \frac{1}{2} \Delta+E_{12}^{s}-E_{12}^{t}$ with the same intensity $\frac{1}{16} C$, the emitted photons of all three last peaks having the linear polarization along the axis $\mathrm{O} y$.

Consider now another example related to the experimental work [3]: the pumping electromagnetic radiation generates the resonant transitions between the ground state of the QD with a non-vanishing biexciton binding energy and the state of the singlet exciton. If $|\lambda / \delta|<<1$ then in the case $E_{12}^{t}>\widetilde{E}_{12}$ the emission spectrum has two peaks at the photon energies $\omega_{0} \pm 2 \sqrt{2}|\lambda|$ with the same intensity $\frac{1}{8} C$, the emitted photons being linearly polarized along the axis $\mathrm{O} x$, while in the case $\widetilde{E}_{22}<E_{12}^{t}<\widetilde{E}_{12}$ there are two peaks at the photon energies $\omega_{0}+\delta \pm \sqrt{2}|\lambda|+E_{12}^{t}-E_{12}^{s}$ with the same intensity $\frac{1}{4} C$ and the linear polarization of the emitted photons along the axis $\mathrm{O} y$. However, if $\lambda$ and $\delta$ have the magnitudes of the same order then other emission spectra would be observed. This is the consequence of the influence of the biexciton on the Rabi oscillations between the exciton and the ground state of the QD. 
Thus we have shown that the photon emission spectra of the two-level semiconductor QDs at the optical resonance regime have various structures depending on the biexciton binding energy and the degree of the asymmetry of the QD as well as on the choice of the resonance frequency. Until now mainly the QDs with the biexciton binding energies in the range from 2,5 meV to 3,5 meV were investigated [7, 14, 32 - 34]. In a recent experiment [38] a QD with the biexciton binding energy $1,2 \mathrm{meV}$ was fabricated. According to the calculations in the theoretical works [22] the biexciton binding energy may be negative. The blueshift of the biexciton luminescence line was also observed [39]. This means that the fabrication of the two-level semiconductor QDs with the biexciton binding energies in a range of several hundreds $\mu \mathrm{eV}$ and with different degrees of asymmetry is possible and would contribute significantly to the experimental study of the optical resonance in the electron system of the QDs.

We have considered the semiconductor QD with the simplest electronic structure. In the reality instead of each bulk semiconductor energy band (conduction band or valence one) there is a set of different discrete energy levels in a semiconductor QD [40 - 45]. Therefore in each semiconductor QD there exist several exciton states called the "bright" excitons with the allowed dipole radiative transitions to its ground state. Although in the QD with the strong electron confinement the influence on the Rabi oscillations of an exciton state by other ones at the exact resonance may be neglected, as we have shown in an example in Sec.3, the existence of many exciton states as well as the presence of the wetting layer might cause a significant change of the photon spontaneous emission spectrum. Moreover usually the spin structures of the wave functions of the discrete energy states in the semiconductor QDs [40 - 45] are more complicated than the simplest one in the model considered in the present work. The Rabi oscillations in the semiconductor QDs with the complicated electronic structures of both abovementioned types will be studied in the subsequent works with the application of the method elaborated in this work.

\section{ACKNOWLEDGEMENTS}

This work was completed in the Max-Planck Institute for the Physics of Complex Systems, Dresden. The authors would like to express their gratitude to Professor Peter Fulde for the interest in this work and the encouragement. We thank MPIPKS for the hospitality, the participants of the Seminar on Electronic Correlations of the Institute for the fruitful discussions, the Vietnamese Academy of Science and Technology and the Vietnam National Natural Sciences Council for the support.

\section{REFERENCES}

1. Allen, L. and Eberly, J.H. (1975), Optical Resonance and Two-Level Atoms, Dover, New York.

2. Stievater, T.H., Li, X., Steel, D.G., Gammon, D., Katzer, D.S., Park, D., Piermarocchi, C., and Sham, L.J. (2001), Phys. Rev. Lett., vol. 87, p. 133603.

3. Kamada, H., Gotoh, H., Temmyo, J., Takagahara, T., and Ando, H. (2001), Phys. Rev. Lett. vol. 87, p. 246401.

4. Htoon, H., Takagahara, T., Kulik, D., Baklenov, O., Holmes, A.Li.Jr., and Shih, C.K. (2002), Phys. Rev. Lett., vol. 88, p. 087401.

5. Besombes, L., Baumberg, J.J., and Motoshita, J. (2003), Phys. Rev. Lett., vol. 90, p. 257402.

6. Förstner, J., Weber, C., Danckwert, J., and Knorr, A. (2003), Phys. Rev. Lett., vol. 91, p. 127401. 
7. Borri, P., Langbein, W., Schneider, S., Woggon, U., Sellin, R.L., Ouyang, D., and Bimberg, D. (2002), Phys. Rev., vol. B 66, p. 081306.

8. Stufler, S., Ester, P., Zenner, A., and Bichler, M. (2005), Phys. Rev., vol. B 72, p. 121301(R).

9. Wang, Q.Q., Muller, A., Bianucci, P., Rossi, E., Xue, Q.K., Takagahara, T., Piermarocchi, C., MacDonald, A.H., and Shih, C.K. (2005), Phys. Rev., vol. B 72, p. 035306.

10. Slepyan, G.Ya., Magyarov, A., Maksimenko, S.A., Hoffmann, A., and Bimberg, D. (2004), Phys. Rev., vol. B 70, p. 045320.

11. Vagov, A., Axt, V.M., and Kuhn, T. (2002), Phys. Rev., vol. B 66, p. 165312.

12. Hohenester, U. (2002), Phys. Rev., vol. B 66, p. 245323.

13. Machnikowski, P. and Jacak, L. (2004), Phys. Rev., vol. B 69, p. 193302.

14. Stufler, S., Machnikowski, P., Ester, P., Bichler, M., Axt, V.M., Kuhn, T., and Zrenner, A. (2006), Phys. Rev., vol. B 73, p. 125304.

15. Vasko, F.T. (2004), Phys. Rev., vol B 70, p. 073305.

16. Axt, V.M., Kuhn, T., Vagov, V., and Peeters, F.M. (2005), Phys. Rev., vol. B 72, p. 125309.

17. Smirnov, A.Yu. and Mouroch, L.G. (2005), Phys. Rev., vol. B 71, p. 161305(R).

18. Villas-Bôas, J.M., Ulloa, S.L., and Govorov, A.O. (2005), Phys. Rev. Lett., vol. 94, p. 057404; cond-mat/0509731, Physica, vol. E 26, p. 337.

19. Unold, T., Mueller, K., Lienau, C., Elsaesser, T., and Wieck, A.D. (2005), Phys. Rev. Lett., vol. 94, p. 137404.

20. Tsukanov, A.V. (2006), Phys. Rev., vol. B 73, p. 085308.

21. Khitrova, G., Gibbs, H.M., Kira, M., Koch, S.W., and Scherer, A. (2006), Nature Physics, vol. 2, p. 81.

22. Kuo, D.M.-T. and Chang, Y.-C. (2003), Phys. Rev., vol. B 67, p. 035313; (2004), vol. B 69, p. 041306(R); (2005), vol. B 72, p. 085334.

23. Haug, H. and Koch, S.W. (1993), Quantum Theory of the Optical and Electronic Properties of Semiconductors, World Scientific, Singapore.

24. Binder, R., Koch, S.W., Lindberg, M., and Peyghambarian, N. (1990), Phys. Rev. Lett., vol. 65, p. 899.

25. Schäfer, W. and Hennenberger, K. (1990), Phys. Status Solidi, vol. B 159, p. 59.

26. Cundiff, S.T., Knorr, A., Feldmann, J., Koch, S.W., Göbel, E.O., and Nickel, H. (1994), Phys. Rev. Lett., vol. 73, p. 1178.

27. Fürst, C., Leitenstorfer, A., Nutsch, A., Tränkle, G., and Zrenner, A. (1997), Phys. Status Solidi (b), vol. 204, p. 20.

28. Schülzgen, A., Binder, R., Donovan, M.E., Lindberg, M., Wundke, K., Gibbs, H.M., Khitrova, G., and Peyghambarian, N. (1999), Phys. Rev. Lett., vol. 82, p. 2346.

29. Rössler, U. (2002), Phys. Status Solidi, vol. B 234, p. 385.

30. Lechner, C. and Rössler, U. (2005), Phys. Rev., vol. B 72, p. $045311 ; 153317$.

31. Chen, P., Piermarocchi, C., and Sham, L.J. (2001), Phys. Rev. Lett., vol. 87, p. 067401. 
32. Kuther, A., Bayer, M., Forchel, A., Gorbunov, A., Timofeev, V.B., Schäfer, F., and Reithmaier, J.P. (1998), Phys. Rev., vol. B 58, p. 7508(R).

33. Langbein, W., Borri, P., Woggon, U., Stavarache, V., Reuter, D., and Wieck, A.D. (2004), Phys. Rev., vol. B 69, p. 161301; vol. B 70, p. 033301.

34. Lenihan, A.S., Gurudev Datt, M-V., Steel, D.G., Ghosh, S., and Bhattacharya, P. (2004), Phys. Rev., vol. B 69, p. 045306; (2002), Phys. Rev. Lett., vol. 88, p. 223601.

35. Bayer, M., Kuther, A., Forchel, A., Gorbunov, A., Timofeev, V.B., Schäfer, F., Reithmaier, J.P., Reinecke, T.L., and Walck, S.N. (1999), Phys. Rev. Lett., vol. 82, p. 1748.

36. Bayer, M., Ortner, G., Forchel, A., Hawrylak, P., and Fafard, S. (2002), Physica, vol. E 13, p. 123.

37. Högele, A., Alen, B., Bickel, F., Warburton, R.J., Petroff, P.M., and Karrai, K. (2004), Physica, vol. E 21, p. 175.

38. Kono, S., Kirihara, A., Tomita, A., Nakamura, K., Fujikata, J., Ohashi, K., Saito, H., and Nishi, K. (2005), Phys. Rev., vol. B 72, p. 155307.

39. Yuan, Z., Kardynal, B.E., Stevenson, R.M., Shields, A.J., Lobo, C.J., Cooper, K., Beattie, N.S., Ritchie, D.A., and Pepper, M. (2002), Science, vol. 295, p. 102.

40. Efros, Al. L. (1992), Phys. Rev., vol. B 46, p. 7448.

41. Norris, D.J. and Badwendi, M.G. (1996), Phys. Rev., vol. B 53, p. 16338.

42. Efros, Al.L., Rosen, M., Kuno, M., Nirmal, M., Norris, D.J., and Badwendi, M.G. (1996), Phys. Rev., vol. B 54, p. 4843.

43. Cortez, S., Krebs, O., Voisin, P., and Gérard, J.M. (2001), Phys. Rev., vol. B 63, p. 233306.

44. Prado, S.J., Trallero-Giner, C., Alcalde, A.M., López-Richard, V., and Marques, G.E. (2003), Phys. Rev., vol. B 68, p. 235327.

45. Li, J. and Xia, J.-B. (2000), Phys. Rev., vol. B 61, p. 15800. 